\title{
High flow nasal cannulae for respiratory support in adult intensive care patients (Protocol)
}

\author{
Corley A, Rickard CM, Aitken LM, Johnston A, Barnett A, Fraser JF
}
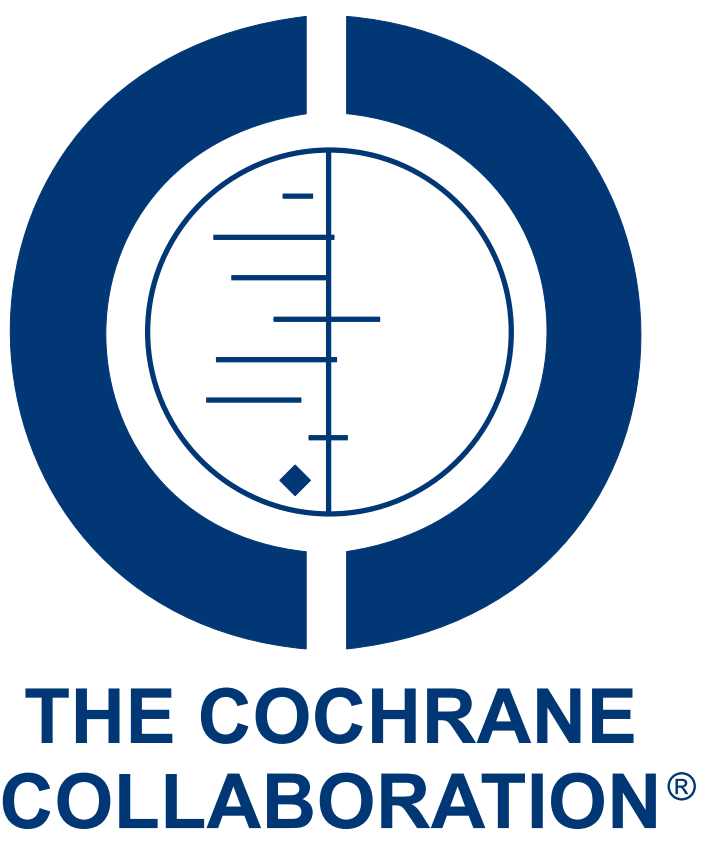

This is a reprint of a Cochrane protocol, prepared and maintained by The Cochrane Collaboration and published in The Cochrane Library 2012, Issue 11

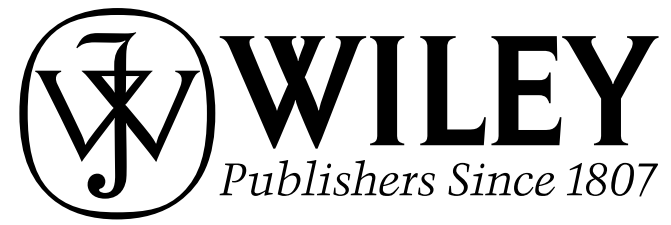

High flow nasal cannulae for respiratory support in adult intensive care patients (Protocol)

Copyright $\odot 2012$ The Cochrane Collaboration. Published by John Wiley \& Sons, Ltd. 
TABLE OF CONTENTS

HEADER . . . . . . . . . . . . . . . . . . . . . . . . . . . . . . . . . . . . . . . . 1

ABSTRACT . . . . . . . . . . . . . . . . . . . . . . . . . . . . . . . . . . . . . . 1

BACKGROUND . . . . . . . . . . . . . . . . . . . . . . . . . . . . . . . . . . . . . 2

OBJECTIVES . . . . . . . . . . . . . . . . . . . . . . . . . . . . . . . . . . . . . . .

METHODS . . . . . . . . . . . . . . . . . . . . . . . . . . . . . . . . . . . . . .

ACKNOWLEDGEMENTS . . . . . . . . . . . . . . . . . . . . . . . . . . . . . . . . . . . . . . . .

REFERENCES . . . . . . . . . . . . . . . . . . . . . . . . . . . . . . . . . . . . . . 7

APPENDICES . . . . . . . . . . . . . . . . . . . . . . . . . . . . . . . . . . . . .

HISTORY . . . . . . . . . . . . . . . . . . . . . . . . . . . . . . . . . . . . . . . . 21

CONTRIBUTIONS OF AUTHORS . . . . . . . . . . . . . . . . . . . . . . . . . . . . . . . . . . 21

DECLARATIONS OF INTEREST . . . . . . . . . . . . . . . . . . . . . . . . . . . . . . . . . . . . . .

SOURCES OF SUPPORT . . . . . . . . . . . . . . . . . . . . . . . . . . . . . . . . . . . .

High flow nasal cannulae for respiratory support in adult intensive care patients (Protocol)

Copyright $\odot 2012$ The Cochrane Collaboration. Published by John Wiley \& Sons, Ltd. 


\title{
[Intervention Protocol]
}

\section{High flow nasal cannulae for respiratory support in adult intensive care patients}

\author{
Amanda Corley ${ }^{1,2}$, Claire M Rickard ${ }^{2}$, Leanne M Aitken ${ }^{3}$, Amy Johnston $^{2}$, Adrian Barnett ${ }^{4}$, John F Fraser ${ }^{1,5}$ \\ ${ }^{1}$ Critical Care Research Group, Intensive Care Services, The Prince Charles Hospital, Chermside, Australia. ${ }^{2}$ School of Nursing \\ and Midwifery, Research Centre for Clinical and Community Practice Innovation, Griffith University, Nathan, Australia. ${ }^{3}$ Research \\ Centre for Clinical and Community Practice Innovation, Griffith University \& Princess Alexandra Hospital, Woolloongabba, Australia. \\ ${ }^{4}$ Institute of Health and Biomedical Innovation, School of Public Health, Queensland University of Technology, Kelvin Grove, Australia. \\ ${ }^{5}$ University of Queensland, St Lucia, Australia
}

Contact address: Amanda Corley, amanda_corley@health.qld.gov.au.

Editorial group: Cochrane Anaesthesia Group.

Publication status and date: New, published in Issue 11, 2012.

Citation: Corley A, Rickard CM, Aitken LM, Johnston A, Barnett A, Fraser JF. High flow nasal cannulae for respiratory support in adult intensive care patients. Cochrane Database of Systematic Reviews 2012, Issue 11. Art. No.: CD010172. DOI: 10.1002/14651858.CD010172.

Copyright (C) 2012 The Cochrane Collaboration. Published by John Wiley \& Sons, Ltd.

\begin{abstract}
A B S T R A C T
This is the protocol for a review and there is no abstract. The objectives are as follows:

We will evaluate studies which include patients 16 years and over admitted to the ICU and who require treatment with HFNC. We will assess the safety and efficacy of HFNC compared with the comparator interventions in terms of failure of treatment, mortality, adverse events, duration of respiratory support, hospital and ICU length of stay, respiratory effects, patient reported outcomes, and cost of treatment.
\end{abstract}

High flow nasal cannulae for respiratory support in adult intensive care patients (Protocol)

Copyright (c) 2012 The Cochrane Collaboration. Published by John Wiley \& Sons, Ltd. 


\section{B A C K G ROU N D}

\section{Description of the condition}

Acute respiratory failure, and the subsequent need for respiratory support, is a frequent cause of admission of adults to an intensive care unit (ICU) (Behrendt 2000). In such patients respiratory support is required due to hypoxaemia, ventilatory failure, or both (Shelly 1999). This respiratory support can be provided to the patient in either an invasive or non-invasive manner.

Invasive mechanical ventilation involves the insertion of an artificial airway (either an endotracheal or tracheostomy tube). Whilst regarded as a life saving treatment, this comes with multiple inherent risks to patients. These risks include the development of ventilator induced lung injury (Gattinoni 2012), ventilator associated pneumonia (Muscadere 2008), neuro-cognitive sequelae associated with prolonged sedation (Morandi 2011; Nelson 2000) and both increased ICU and hospital length of stay (Safdar 2005). Where possible, therefore, invasive mechanical ventilation should be avoided, however intubation and mechanical ventilation are inevitable if the patient has stopped breathing or is unable to maintain his or her airway (Nava 2009).

Non-invasive respiratory support, where possible, is the preferred method of respiratory support and can be delivered via any of the following equipment:

- low flow nasal cannulae (LFNC);

- simple face mask;

- venturi mask;

- non-rebreather mask;

- non-invasive positive pressure ventilation (NIPPV); or

- high flow nasal cannulae (HFNC).

The type of delivery device chosen is largely dependent on the severity and aetiology of the patient's acute respiratory failure, with each device having benefits and drawbacks which determine their usefulness in clinical practice.

LFNC are used for patients requiring a small amount of respiratory support in the form of supplemental oxygen to maintain adequate oxygenation. They deliver dry oxygen at one to six litres per minute via small prongs approximately $1.5 \mathrm{~cm}$ in length which sit just inside the nares of the patient's nose (O'Driscoll 2008). Although they are generally well tolerated by patients (Zevola 2001), the delivery of higher flows of oxygen through low flow nasal cannulae is not practicable due to the drying and irritating effects of the cold dry gas on the mucosa (Lellouche 2002).

The delivery of oxygen via a face mask is necessary if the patient has higher oxygen requirements than can be achieved with LFNC. Simple face masks are used to deliver five to 10 litres per minute of oxygen. For those patients requiring more oxygen and higher flows to maintain adequate oxygenation, non-rebreather masks can be used to deliver 10 to 15 litres per minute of oxygen (O'Driscoll 2008). Oxygen may be supplemented with humidification by some devices. Simple face masks and non-rebreather masks are capable of delivering relatively high oxygen concentrations, therefore they are generally unsuitable for patients with chronic obstructive pulmonary disease (COPD) who may retain carbon dioxide. For hypercapnoeic COPD patients, the oxygen concentration can be regulated by using a venturi mask which can deliver between $24 \%$ and $60 \%$ oxygen at a flow of two to 15 litres per minute (O'Driscoll 2008). Although face masks are effective at delivering oxygen to patients with mild to moderate acute respiratory failure, they can be poorly tolerated when compared with nasal cannulae due to discomfort and feelings of claustrophobia. This can lead to reduced compliance as a result of frequent removal and subsequent treatment interruption (Sasaki 2003).

NIPPV is used in patients who not only require supplemental oxygen but also need support of the mechanical process of ventilation (Mehta 2001). Blended oxygen and air is delivered at a prescribed fraction of inspired oxygen $\left(\mathrm{FiO}_{2}\right)$ via a tight fitting mask (nasal mask, oronasal mask or full face mask). Additionally, either continuous positive airway pressure (CPAP) or bilevel positive airway pressure ventilation (BiPAP) is delivered to improve alveolar recruitment, improve gas exchange and decrease the work of breathing (Mehta 2001). Whilst CPAP is not a true ventilatory mode it is often referred to as NIPPV in clinical practice (Nava 2009). Substantial data are available showing that NIPPV improves outcomes in patients requiring respiratory support due to cardiogenic pulmonary oedema or acute exacerbations of COPD and also for patients weaning from invasive mechanical ventilation (Nava 2009). However, its relevance to patients with hypoxaemic acute respiratory failure is less clearly defined (Nava 2009). Despite showing clear benefits in certain conditions, NIPPV inhibits mobilization, is associated with gastric distension, restricts effective communication and oral nutrition, and is poorly tolerated by some patients due to discomfort (Gregoretti 2002; Mehta 2001). While the conventional non-invasive delivery devices listed above are important therapies in the range of respiratory support therapies available to treat acute respiratory failure, it is evident that they have limitations which can impact on their usefulness in clinical practice. The failure of these devices to provide adequate respiratory support and to correct the acute respiratory failure often results in the patient requiring intubation and mechanical ventilation.

\section{Description of the intervention}

HFNC, which have been used in the neonatal setting for some years (Wilkinson 2011), are a relatively new method of delivering respiratory support to adults experiencing acute respiratory failure. The cannulae are approximately $1.5 \mathrm{~cm}$ in length, $0.5 \mathrm{~cm}$ in diameter and, as with LFNC, sit just inside the nares. A gas flow of up to 60 litres per minute can be delivered because the gas is warmed and humidified making it less irritant to the nasal mucosa. For the purposes of this review, HFNC will be defined as humidified oxygen delivered via nasal cannulae at a rate greater 
than 20 litres per minute. Very few adverse reactions have been reported with HFNC use and, of those reported, they consist of minor complaints of a runny nose (Price 2008) and some discomfort with the heat or flow rate (Roca 2010).

\section{How the intervention might work}

HFNC deliver blended humidified air and oxygen via wide bore nasal cannulae at a prescribed fraction of inspired oxygen $\left(\mathrm{FiO}_{2}\right)$ at high flow rates. HFNC do not need to be removed during oral hygiene care or when the patients talk, eat or drink, resulting in less frequent interruptions to therapy. In the growing body of evidence investigating the effects of HFNC, improvements in oxygenation (Corley 2011; Parke 2009; Roca 2010; Sztrymf 2011 Nov; Sztrymf 2011), respiratory rate (Corley 2011; Roca 2010; Sztrymf 2011 Nov; Sztrymf 2011), dyspnoea (Corley 2011; Roca 2010; Sztrymf 2011 Nov) and patient comfort (Corley 2011; Roca 2010; Tiruvoipati 2010) have been reported in recent observational studies.

The suggested mechanisms of action of HFNC are:

- flushing of anatomical dead space due to the high gas flow thereby functionally reducing dead space and improving respiratory efficiency (Dysart 2009);

- generation of positive airway pressure (Corley 2011; Groves 2007; Parke 2009), which increases functional residual capacity and improves alveolar recruitment;

- the ability to better meet the high inspiratory flow demands seen in patients requiring respiratory support and to deliver a more accurate $\mathrm{FiO}_{2}$ through less dilution by entrainment of room air (Dysart 2009);

- the ability to deliver optimal humidification leading to enhanced mucociliary transport (Salah 1988) and improved patient comfort (Chanques 2009).

The purpose of this review is to compare the efficacy and safety of HFNC with other methods of non-invasive respiratory support in adult patients admitted to the ICU.

\section{Why it is important to do this review}

Whilst it has been demonstrated that HFNC offer some immediate physiological benefits to patients requiring respiratory support, it has yet to be determined whether they offer any clinically important benefits and improve patient outcomes, such as preventing progression to invasive mechanical ventilation and reducing mortality. Individual studies may tend to focus on surrogate outcomes or be underpowered to detect effects on clinically important outcomes. By performing this review we will be able to extract data on important clinical outcomes and, through meta-analysis, analyse the effects of the intervention on these with greater statistical power to detect meaningful patient differences should they exist.
As HFNC gain in popularity as a treatment modality for providing respiratory support, it is important to perform this review to synthesize the existing evidence base and to provide clear conclusions regarding the efficacy and safety of HFNC. In this way, clinicians can make decisions about how this form of respiratory support can best be incorporated into the current suite of treatment options; and in whom the treatment could be used most efficaciously.

\section{O B J E C T I VES}

We will evaluate studies which include patients 16 years and over admitted to the ICU and who require treatment with HFNC. We will assess the safety and efficacy of HFNC compared with the comparator interventions in terms of failure of treatment, mortality, adverse events, duration of respiratory support, hospital and ICU length of stay, respiratory effects, patient reported outcomes, and cost of treatment.

\section{METHODS}

\section{Criteria for considering studies for this review}

\section{Types of studies}

We will include all randomized, parallel and quasi-randomized studies (including crossover studies) which compare HFNC to other forms of non-invasive respiratory support for the selected outcome measures. We will include quasi-randomized trials in this review due to the current scarcity of randomized trials in this area. Due to the inability of randomized crossover studies to detect long term patient outcomes, we will only include this trial design for the secondary outcome measures of positive end expiratory pressure, oxygenation, carbon dioxide clearance, respiratory rate, work of breathing, and patient reported outcomes.

We will not impose a language restriction and we will include studies written in any language.

We will exclude retrospective studies and prospective cohort or observational studies as we want to focus on the highest quality evidence from randomized studies.

\section{Types of participants}

We will include studies which enrol adult patients (16 years or over) requiring respiratory support and admitted to the intensive care unit (ICU).

We will exclude participants aged less than 16 years. A Cochrane review is already published assessing the effectiveness of HFNC in preterm infants (Wilkinson 2011) and at present there is a protocol 
in progress for a systematic review with the Cochrane Anaesthesia Group assessing HFNC effectiveness in the paediatric population (Mayfield 2012). We will also exclude patients not admitted to an ICU.

\section{Types of interventions}

We will include humidified oxygen delivered via the nasal route at a rate greater than 20 litres per minute as the experimental intervention.

We will include the following forms of non-invasive respiratory support as comparison interventions:

- low flow oxygen via nasal cannulae or mask (15 litres per minute or less);

- continuous positive airway pressure (CPAP);

- bilevel positive airway pressure (BiPAP).

\section{Types of outcome measures}

The outcome measures in this review are a mix of surrogate and clinical outcomes. We recognise that while there may be a correlation between the surrogate and clinical outcomes, it is the clinical outcomes which will provide the strongest evidence regarding the safety and efficacy of HFNC. As a result, we have chosen the clinical outcome of failure of treatment as indicated by the need for NIPPV or invasive ventilation as one of our primary outcome measures.

\section{Primary outcomes}

1. Failure of treatment as indicated by the need for NIPPV or invasive ventilation (up to 28 days)

2. In hospital mortality (up to 90 days)

3. Adverse events

\section{Secondary outcomes}

1. Duration in hours of any form of respiratory support (mechanical ventilation, NIPPV, HFNC, standard oxygen).

2. Length of stay in days (ICU and hospital).

3. Respiratory effects as indicated by any of the following:

- degree of atelectasis on radiological examination;

- positive end-expiratory pressure measured at the pharyngeal level $\left(\mathrm{cm} \mathrm{H}_{2} \mathrm{O}\right)$;

- oxygenation $\left(\mathrm{PaO}_{2} / \mathrm{FiO}_{2}\right.$ ratio, $\mathrm{PaO}_{2}, \mathrm{SaO}_{2}$ and $\left.\mathrm{SpO}_{2}\right)$;

- carbon dioxide clearance $\left(\mathrm{PaCO}_{2}\right.$ and $\left.\mathrm{pCO}_{2}\right)$;

- respiratory rate;

- work of breathing (joules per litre).

4. Patient reported outcomes as indicated by any of the following:

- dyspnoea;

- comfort;

- mouth dryness;

- patient refusal to continue with treatment.
5. Cost comparison of treatment (in Australian dollars).

All outcomes will be assessed at the time points reported in the included studies. For patient reported outcomes, we will accept the study authors' definitions.

\section{Search methods for identification of studies}

\section{Electronic searches}

We will search the following databases:

- Cochrane Central Register of Controlled Trials

(CENTRAL) in The Cochrane Library (most recent issue);

- MEDLINE, OvidSP (January 2000 to date);

- CINAHL, EBSCOhost (January 2000 to date);

- EMBASE, OvidSP (January 2000 to date);

- ISI Web of Science (January 2000 to date).

The search start date has been restricted to 2000 as HFNC have only been available for use in the adult population since the mid2000s. The detailed search strategy for MEDLINE is available in Appendix 1. We will adapt this search strategy for searching all other databases.

We will search for published abstracts from conference proceedings for the European Society of Intensive Care Medicine, the Australia and New Zealand Intensive Care Society, the Society of Critical Care Medicine and the American Thoracic Society (2000 to date). For trials not yet completed, we will search clinical trial registries (clinicaltrials.gov; controlled-trials.com; anzctr.org.au and who.int/ictrp). We (AC) will contact the trial authors to determine if any data are available for inclusion in the review.

\section{Searching other resources}

We will screen the reference lists of eligible trials to identify any previously unidentified studies.

\section{Data collection and analysis}

\section{Selection of studies}

We will include in the review all randomized, parallel group, and quasi-randomized controlled trials meeting the criteria. Two authors (AC and CR) will independently examine the published titles and abstracts obtained from the search strategy and screen them for suitability. A study selection form (see Appendix 2) will be completed by each author and if the study is to be excluded we will detail the reasons for exclusion. Any disagreement will be resolved by LA. If any studies for which $\mathrm{AC}, \mathrm{AB}$ or JF are listed as authors are contained within the search results, CR and LA will perform the screening for suitability with AJ resolving any disagreement.

High flow nasal cannulae for respiratory support in adult intensive care patients (Protocol) 


\section{Data extraction and management}

Two authors (AC and CR) will independently extract data from each study onto the data extraction form (see Appendix 3). Data extraction forms for each study will be compared. We will resolve any disagreement by discussion with LA. If data are being extracted from any studies on which $\mathrm{AC}, \mathrm{AB}$ or JF are listed as authors, $\mathrm{CR}$ and LA will perform the data extraction with $\mathrm{AJ}$ resolving any disagreement.

\section{Assessment of risk of bias in included studies}

Two authors (AC and CR) will independently assess the risk of bias in each of the studies. We will resolve any disagreements by discussion with LA. We (AC) will contact trial authors if more information is required to make an assessment on risk of bias. If the risk of bias is being assessed in any studies in which $\mathrm{AC}, \mathrm{AB}$ or JF are listed as authors, CR and LA will perform the assessment with $\mathrm{AJ}$ resolving any disagreement.

We will conduct the assessment of risk of bias in included studies using the 'Risk of bias' tool as per the Cochrane Handbook for Systematic Reviews of Interventions (Higgins 2011), using the quality assessment form (see Appendix 4). We will assess trials as having a low risk of bias if all of the following areas are assessed as adequate. We will assess trials as having a high risk of bias if one or more of the areas are assessed as not adequate or unclear.

We will assess the risk of bias in the following domains.

1. Random sequence generation (selection bias)

We will assess allocation of interventions as adequate if allocation was performed in a truly unpredictable manner (for example computerized random number generator, random number table, shuffled envelope system, coin toss or roll of the die).

We will assess allocation as inadequate if it is based on non-random methods (for example day of the week, alternate patients, patient characteristics such as date of birth or hospital identifier) or the method of allocation is unclear.

2. Allocation concealment (selection bias)

We will assess allocation concealment as adequate if study personnel and participants were unaware of the treatment allocation of the next participant (for example central or telephone randomization, sequentially-numbered sealed opaque envelopes, on-site computer accessed only after patient enrolment).

Inadequate allocation concealment will include those randomization methods deemed inadequate in point 1 (for example unsealed or non-sequential envelopes, open allocation sequence) or if it is unclear what method of allocation concealment was used.

3. Blinding of outcome assessors (performance and detection bias) It is not possible to blind the participant or the clinical staff to the treatment allocation for this intervention, therefore we will limit risk of bias to the blinding of outcome assessors.

We will assess blinding as adequate where outcome assessors are definitely blinded to the treatment allocation. If there is no mention of blinding, we will deem blinding as not adequate.
Where blinding of outcome assessors is not always possible (for example respiratory rate, oxygenation, carbon dioxide clearance, work of breathing) we will assess whether this would have been likely to have introduced bias. If measurement of the outcome is unlikely to have been influenced by the lack of blinding, adequate blinding will be assumed.

Where the patient is the outcome assessor (that is for patient reported outcomes) we will deem the blinding adequate if there is evidence of strategies to reduce potential bias (for example a standardised questioning strategy used for all patient reported outcomes, questioning carried out by non-study staff).

4. Incomplete outcome data, intention to treat (attrition bias) We will deem outcome data as adequate if all withdrawals, protocol deviations and losses to follow-up are reported and the incomplete data are proportionate across groups. If this is not reported, we will assess outcome data as being inadequately dealt with.

5. Selective reporting

We will assess outcome reporting as adequate if all previously stated outcomes have been fully reported. We will assess outcome reporting as inadequate if: all previously stated outcomes have not been reported, if outcomes are not fully reported, or if outcomes are reported but were not previously mentioned.

\section{Measures of treatment effect}

We will perform statistical analyses using Review Manager 5 (RevMan 5.1) and R (www.r-project.org). We will express dichotomous data as risk ratio (RR), risk difference (RD) and number needed to treat (NNT). We will express continuous data as the difference between the means. We will report the $95 \%$ confidence interval (CI) for all estimates. Ordinal data (for example mouth dryness on a scale of 0 to 10 ) will be treated as continuous data. To cope with any non-normally distributed data, we will use the generalised linear model framework assuming a gamma distribution.

\section{Unit of analysis issues}

For parallel trials, we will aim to only include studies where the unit of analysis is the patient. Our meta-analysis will be based on summary statistics (for example odds ratios) derived from patient level data.

For crossover trials, we will include only the outcomes from the first treatment period and treat the data as per a parallel group trial. If the data in crossover trials are not reported as per patient for the first treatment period, then we will request this data from the authors.

\section{Dealing with missing data}

We will identify the number of missing data points for each study. If these are not explicit, the trial author will be contacted. The methods used to account for missing data will be assessed on a case-by-case basis. 
Missing data have the potential to bias the study: by hiding unfavourable outcomes (for example a participant who did not tolerate the treatment), or by varying between treatments (for example less missing data for the control group). We will tabulate the percent of missing data for the primary and secondary outcomes by treatment for each study. We will use sensitivity analyses to check the influence of missing data. We will drop studies from a sensitivity analysis if more than $20 \%$ of the primary or secondary outcomes are missing, or if the rate of those missing in the HFNC group is half or double the rate in any other group (with a minimum rate of $5 \%)$.

\section{Assessment of heterogeneity}

Using clinical judgement, we will assess participants, interventions and outcomes for clinical heterogeneity. We will assess methodological heterogeneity during the risk of bias assessments and by visual inspection of forest plots. We will assess statistical heterogeneity using the $\mathrm{I}^{2}$ statistic and $\mathrm{Chi}^{2}$ test (on a scale of $0 \%$ to 100\%) (Higgins 2011).

\section{Assessment of reporting biases}

If more than 10 trials are available for the meta-analysis, we will assess publication bias by visually inspecting the symmetry of the funnel plot.

\section{Data synthesis}

Sequential meta-analysis will be used for the primary outcome of treatment failure only, as that is the key variable on which the success of the treatment is judged (that is the stopping rule) and hence the variable for which we want to avoid type I errors associated with multiple testing (see 'Sequential analysis' section below). The sequential meta-analysis recommended by Higgins and Green (Higgins 2011) is a random-effects model analysis and there is no fixed-effect analogue. Higgins and Green (Higgins 2011) specifically warned against using a fixed-effect approach for sequential meta-analysis, and their simulations showed good coverage for their sequential random-effects analysis when there was no heterogeneity. Hence we will not use a fixed effect approach for the primary variable.

For the secondary outcomes, we will conduct the meta-analysis using a fixed-effect model if the studies selected for inclusion do not have significant heterogeneity. The level of heterogeneity will be classified using the $\mathrm{I}^{2}$ statistic as: $0 \%$ to $40 \%$, not important; $30 \%$ to $60 \%$, moderately important; $50 \%$ to $90 \%$, substantially important; and $>90 \%$, considerably important (Higgins 2002). If heterogeneity is assessed as being significant, we will use a randomeffects model. We will conduct analyses for secondary outcomes using Review Manager 5 (RevMan 5.1).

\section{Sequential analysis}

As this an emerging field there is a potential for much new evidence in the coming years, which means we may update the meta-analysis often. Repeating the meta-analysis increases the chance of a type I error. To overcome this issue we will use the approximate semiBayes approach (Higgins 2011a). This method uses monitoring boundaries, which are used to assess: i) sufficient evidence of an effect, or ii) sufficient evidence of the lack of an effect. The method is based on a pre-specified significance level, power, and a clinically important effect. We have chosen a significance level of 0.05 , a power of 0.95 , and a clinically important effect of $\log (0.4)$ for failure of treatment. This clinically important effect is based on the average odds ratio from three similar studies. This gives the boundaries of $Z=9.0$ (upper and lower boundaries) and $V_{\max }=9.9$ (vertical boundary). The $Z$ boundary is used to assess the statistical significance for each sequential meta-analysis. The $V$ boundary gives the maximum amount of information. So a study size beyond this $V$ boundary with no results outside the $Z$ boundaries means we can accept the null hypothesis.

The Higgins et al (Higgins 2011a) method applies a Bayesian prior distribution to the heterogeneity. This is particularly useful when there are few trials in the meta-analysis, so that a more realistic estimate of the heterogeneity is used in the random effects model meta-analysis. We will use an inverse gamma prior for the heterogeneity $\left(\tau^{2}\right)$ with a shape parameter of 2.6 and scale parameters of 0.4 . This is based on a simulation study using randomly generated groups of studies, of between two and 19 available studies, with randomly generated sample sizes between 15 and 990 per group and random treatment effects centred on a log odds ratio of 0.4 . All these simulation choices were informed by similar studies in the field. We will re-visit this prior distribution before running the sequential meta-analysis to assess whether it can be updated based on the most recent evidence.

We will use the $\mathrm{R}$ code (Higgins 2011a) to run the sequential meta-analysis. Forest and funnel plots will also be created in $\mathrm{R}$ using the "rmeta" package (Lumley 2009).

\section{Subgroup analysis and investigation of heterogeneity}

We will perform a subgroup analysis for the participant group and intervention if sufficient detail is present in the eligible studies.

\section{Participants}

- Age $\geq 65$ years.

- Body mass index (BMI) $\geq 30 \mathrm{~kg} / \mathrm{m}^{2}$.

- Aetiology of acute respiratory failure (Type 1 (hypoxia without hypercapnia) versus Type 2 (hypoxia with hypercapnia) respiratory failure).

- Acute respiratory failure versus post-extubation patients.

- Obstructive sleep apnoea.

High flow nasal cannulae for respiratory support in adult intensive care patients (Protocol) 


\section{Intervention}

- HFNC flow rate:

- less than 40 litres per minute,

- 40 to 50 litres per minute,

- more than 50 litres per minute.

\section{Sensitivity analysis}

To determine the sensitivity of the findings to the way in which we have conducted the analysis, we will perform a sensitivity analysis in the following areas.

- Trials with unclear or low risk of bias versus high risk of bias.

- Randomized versus quasi-randomized trials.

- A random-effects model versus fixed-effect model (for secondary outcomes only).

- Excluding trials with relatively large amounts of missing data (more than $10 \%$ of data missing for each outcome variable).

\section{Summary of findings}

We will use the principles of the GRADE system (Guyatt 2008) to assess the quality of the body of evidence associated with the specific outcomes listed below.

- Failure of treatment as indicated by the need for NIPPV or invasive ventilation.

- Duration in hours of any form of respiratory support (mechanical ventilation, NIPPV, HFNC, standard oxygen).
- Length of stay in days (ICU and hospital).

- Atelectasis.

- Oxygenation.

- Carbon dioxide clearance.

- Patient reported outcomes.

We will construct a 'Summary of findings' (SoF) table using the GRADE software. The GRADE approach appraises the quality of a body of evidence based on the extent to which one can be confident that an estimate of effect or association reflects the item being assessed. The quality of a body of evidence considers within study risk of bias (methodologic quality), the directness of the evidence, heterogeneity of the data, precision of effect estimates and risk of publication bias.

\section{ACKNOWLEDGEMENTS}

We would like to thank Karen Hovhannisyan for developing the search strategy, and Jane Cracknell for assistance with protocol development. We would also like to thank Harald Herkner (content editor), Cathal Walsh (statistical editor), Georgine Imberger, JeanDamien Ricard and Kevin Dysart (peer reviewers) for their help and editorial advice during the preparation of this protocol for the systematic review.

\section{R E F E R E N C E S}

\section{Additional references}

\section{Behrendt 2000}

Behrendt CE. Acute respiratory failure in the United States: incidence and 31-day survival. Chest 2000 Oct;118(4): 1100-5. [PUBMED: 11035684]

\section{Chanques 2009}

Chanques G, Constantin JM, Sauter M, Jung B, Sebbane M, Verzilli D, et al.Discomfort associated with underhumidified high-flow oxygen therapy in critically ill patients. Intensive Care Medicine 2009 Jun;35(6):996-1003. [PUBMED: 19294365]

\section{Corley 2011}

Corley A, Caruana LR, Barnett AG, Tronstad O, Fraser JF. Oxygen delivery through high-flow nasal cannulae increase end-expiratory lung volume and reduce respiratory rate in post-cardiac surgical patients. British Journal of Anaesthesia 2011 Dec;107(6):998-1004. [PUBMED: 21908497]

Corley, in progress

Corley A, Bull T, Barnett A, Spooner A, Fraser J. Direct extubation onto high flow nasal prongs post cardiac surgery versus standard treatment in patients with a $\mathrm{BMI} \geq 30$ : $\mathrm{A}$ randomised controlled trial. ACTRN12610000942055. In progress.

\section{Dysart 2009}

Dysart K, Miller TL, Wolfson MR, Shaffer TH. Research in high flow therapy: mechanisms of action. Respiratory Medicine 2009 Oct;103(10):1400-5. [PUBMED: 19467849]

Gattinoni 2012

Gattinoni L, Carlesso E, Langer T. Towards ultraprotective mechanical ventilation. Current Opinion in Anaesthesiology 2012;25(2):141-7. [PUBMED: 22246461]

Gregoretti 2002

Gregoretti C, Confalonieri M, Navalesi P, Squadrone V, Frigerio P, Beltrame F, et al.Evaluation of patient skin breakdown and comfort with a new face mask for noninvasive ventilation: a multi-center study. Intensive Care Medicine 2002 Mar;28(3):278-84. [PUBMED: 11904656 ]

\section{Groves 2007}

Groves N, Tobin A. High flow nasal oxygen generates positive airway pressure in adult volunteers. Australian Critical Care 2007 Nov;20(4):126-31. [PUBMED: 17931878]

\section{Guyatt 2008}

Guyatt GH, Oxman AD, Kunz R, Vist GE, Falck-Ytter Y, Schunemann HJ. What is "quality of evidence" and why is 
it important to clinicians?. BMJ 2008 May 3;336(7651): 995-8. [PUBMED: 18456631]

\section{Higgins 2002}

Higgins JP, Thompson SG. Quantifying heterogeneity in a meta-analysis. Statistics in Medicine 2002;21(11):1539-58. [PUBMED: 12111919]

Higgins 2011

Higgins JPT, Green S (editors). Cochrane Handbook for Systematic Reviews of Interventions Version 5.1.0 [updated March 2011]. The Cochrane Collaboration, 2011. Available from www.cochrane-handbook.org.

Higgins 2011a

Higgins JP, Whitehead A, Simmonds M. Sequential methods for random-effects meta-analysis. Statstics in Medicine 2011;30(9):903-21. [PUBMED: 21472757]

Lellouche 2002

Lellouche F, Maggiore SM, Deye N, Taille S, Pigeot J, Harf A, et al.Effect of the humidification device on the work of breathing during noninvasive ventilation. Intensive Care Medicine 2002 Nov;28(11):1582-9. [PUBMED: 12415444]

Lumley 2009

Lumley T. rmeta: Meta-analysis. $\mathrm{R}$ package version 2.16. http://CRAN.R-project.org/package=rmeta (accessed 21 June 2012) 2009.

Mayfield 2012

Mayfield S Jauncey-Cooke, Hough, JL Schibler, A Gibbons K, Bogossian F. High flow nasal cannula therapy for respiratory support in children. Cochrane Database of Systematic Reviews 2012, Issue 5. [DOI: 10.1002/ 14651858.CD009850]

\section{Mehta 2001}

Mehta S, Hill NS. Noninvasive ventilation. American Journal of Respiratory and Critical Care Medicine 2001 Feb; 163(2):540-77. [PUBMED: 11179136]

\section{Morandi 2011}

Morandi A, Brummel NE, Ely EW. Sedation, delirium and mechanical ventilation: the 'ABCDE' approach. Current Opinion in Critical Care 2011 Feb;17(1):43-9. [PUBMED: 21169829]

\section{Muscadere 2008}

Muscedere J, Dodek P, Keenan S, Fowler R, Cook D, Heyland D. Comprehensive evidence-based clinical practice guidelines for ventilator-associated pneumonia: Diagnosis and treatment. Journal of Critical Care 2008;23:138-47. [PUBMED: 18359431]

Nava 2009

Nava S, Hill N. Non-invasive ventilation in acute respiratory failure. Lancet 2009 Jul 18;374(9685):250-9. [PUBMED: 19616722]

Nelson 2000

Nelson BJ, Weinert CR, Bury CL, Marinelli WA, Gross CR. Intensive care unit drug use and subsequent quality of life in acute lung injury patients. Critical Care Medicine 2000 Nov;28(11):3626-30. [PUBMED: 11098964]

\section{O'Driscoll 2008}

O'Driscoll BR, Howard LS, Davison AG. BTS guideline for emergency oxygen use in adult patients. Thorax 2008 Oct;63 Suppl 6:vi1-68. [PUBMED: 18838559]

Parke 2009

Parke R, McGuinness S, Eccleston M. Nasal highflow therapy delivers low level positive airway pressure. British Journal of Anaesthesia 2009 Dec;103(6):886-90. [PUBMED: 19846404]

\section{Price 2008}

Price AM, Plowright C, Makowski A, Misztal B. Using a high-flow respiratory system (Vapotherm) within a high dependency setting. Nursing and Critical Care Nov-Dec 2008;13(6):298-304. [PUBMED: 19128313]

RevMan 5.1

The Nordic Cochrane Centre, The Cochrane Collaboration. Review Manager (RevMan). 5.1. Copenhagen: The Nordic Cochrane Centre, The Cochrane Collaboration, 2011.

Roca 2010

Roca O, Riera J, Torres F, Masclans JR. High-flow oxygen therapy in acute respiratory failure. Respiratory Care 2010 Apr;55(4):408-13. [PUBMED: 20406507]

\section{Safdar 2005}

Safdar N, Dezfulian C, Collard HR, Saint S. Clinical and economic consequences of ventilator-associated pneumonia: a systematic review. Critical Care Medicine 2005 Oct;33 (10):2184-93. [PUBMED: 16215368]

\section{Salah 1988}

Salah B, Dinh Xuan AT, Fouilladieu JL, Lockhart A, Regnard J. Nasal mucociliary transport in healthy subjects is slower when breathing dry air. European Respiratory Journal 1988 Oct;1(9):852-5. [PUBMED: 3229484]

\section{Sasaki 2003}

Sasaki H, Yamakage M, Iwasaki S, Mizuuchi M, Namiki A. Design of oxygen delivery systems influences both effectiveness and comfort in adult volunteers. Canadian Journal of Anaesthesia 2003 Dec;50(10):1052-5. [PUBMED: 14656787]

\section{Shelly 1999}

Shelly MP, Nightingale P. ABC of intensive care: respiratory support. BMJ 1999 Jun 19;318(7199):1674-7. [PUBMED: 10373174]

\section{Sztrymf 2011}

Sztrymf B, Messika J, Mayot T, Lenglet H, Dreyfuss D, Ricard JD. Impact of high-flow nasal cannula oxygen therapy on intensive care unit patients with acute respiratory failure: A prospective observational study. Journal of Critical Care 2012;27(3):e9-13. [PUBMED: 21958974]

\section{Sztrymf 2011 Nov}

Sztrymf B, Messika J, Bertrand F, Hurel D, Leon R, Dreyfuss D, et al.Beneficial effects of humidified high flow nasal oxygen in critical care patients: a prospective pilot study. Intensive Care Medicine 2011 Nov;37(11):1780-6. [PUBMED: 21946925] 
Tiruvoipati 2010

Tiruvoipati R, Lewis D, Haji K, Botha J. High-flow nasal oxygen vs high-flow face mask: a randomized crossover trial in extubated patients. Journal of Critical Care 2010 Sep;25 (3):463-8. [PUBMED: 19781896]

Wilkinson 2011

Wilkinson D, Andersen C, O'Donnell CPF, De Paoli AG. High flow nasal cannula for respiratory support in preterm infants. Cochrane Database of Systematic Reviews 2011, Issue 5. [DOI: 10.1002/14651858.CD006405.pub2]

Zevola 2001

Zevola DR, Maier CB. Use of nasal cannula versus face mask after extubation in patients after cardiothoracic surgery. Critical Care Nurse 2001 Jun;21(3):47-53. [PUBMED: 11858676]

* Indicates the major publication for the study

\section{A P P E N D I C E S}

\section{Appendix I. Search strategy for MEDLINE (OvidSP)}

1 (((high?flow or nasal*) adj6 can?ul*) or HFNC or (nasal adj6 (high?flow or prong)) or Vapotherm or Optiflow).af.

2 ((randomized controlled trial or controlled clinical trial).pt. or randomized.ab. or placebo.ab. or drug therapy.fs. or randomly.ab. or trial.ab. or groups.ab.) not (animals not (humans and animals)).sh.

31 and 2

\section{Appendix 2. Study Selection Form}

\section{Study Details}

First Author

Journal / Place of publication

Year

\begin{tabular}{l|l} 
Study Eligibility Comments & Com
\end{tabular}

Study Type Yes / No / Unclear

- $\quad$ RCT

- $\quad$ Randomized crossover

Relevant participants

Yes / No / Unclear

- $\quad$ Age $\geq 16$ years

- $\quad$ Admitted to Intensive Care

High flow nasal cannulae for respiratory support in adult intensive care patients (Protocol)

Copyright $\odot 2012$ The Cochrane Collaboration. Published by John Wiley \& Sons, Ltd. 
Unit

Relevant interventions
- HFNC compared

with comparison interventions

(LFNC, face mask, CPAP, BiPAP)

Relevant outcomes

Yes / No / Unclear

Failure of treatment as indicated

by the need for NIPPV or invasive

ventilation (up to 28 days)

In hospital mortality (up to 90 days)

Adverse events

Duration in hours of any form of respiratory support (mechanical ventilation, NIPPV, HFNC, standard oxygen)

Length of stay in days (ICU and hospital)

Respiratory effects as indicated by any of the following:

- Degree of atelectasis on radiological examination

- Positive end expiratory pressure measured at the pharyngeal level $\left(\mathrm{cmH}_{2} \mathrm{O}\right)$

- Oxygenation $\left(\mathrm{PaO}_{2} / \mathrm{FiO}_{2}\right.$ ratio, $\mathrm{PaO}_{2}, \mathrm{SaO}_{2}$ and $\mathrm{SpO}_{2}$ )

- Carbon dioxide clearance $\left(\mathrm{PaCO}_{2}\right.$ and $\left.\mathrm{pCO}_{2}\right)$

- Respiratory rate

- Work of breathing (joules per litre)

Patient reported outcomes as indicated by any of the following:

- Dyspnoea

- Comfort

- Mouth dryness

- Patient refusal to continue with treatment Cost comparison of treatment (in Australian dollars) 


\section{Appendix 3. Data Extraction Form}

\section{Data extraction form}

Review title or ID

Study ID (surname of first author and year first full report of study was published e.g. Smith 2001)

Report IDs of other reports of this study (e.g. duplicate publications, follow-up studies)

\section{General Information}

Date form completed $(d d / m m / y y y y)$

Name of person extracting data

Report title

(title of paper/ abstract/ report that data are extracted from)

Report ID

(ID for this paper/ abstract/ report)

Reference details

Report author contact details

\section{Publication type}

(full report, abstract, letter)

\section{Study funding sources}

(including role of funders)

Possible conflicts of interest

(for study authors)

\section{Notes:}

Copyright $\odot 2012$ The Cochrane Collaboration. Published by John Wiley \& Sons, Ltd. 


\section{Population and setting}

\begin{tabular}{|c|c|c|}
\hline & $\begin{array}{l}\text { Description } \\
\text { Include comparative information for each } \\
\text { group (i.e. intervention and controls) if } \\
\text { available }\end{array}$ & $\begin{array}{l}\text { Location in text } \\
(p g \text { \&fg/table })\end{array}$ \\
\hline \multicolumn{3}{|l|}{$\begin{array}{l}\text { Population description } \\
\text { (from which study participants } \\
\text { are drawn) }\end{array}$} \\
\hline \multicolumn{3}{|l|}{$\begin{array}{l}\text { Setting } \\
\text { Including: } \\
\cdot \text { Country of study } \\
\cdot \quad \text { Level of Hospital (Tertiary, } \\
\text { Metropolitan, Regional, Rural) } \\
\cdot \quad \text { Number of beds }\end{array}$} \\
\hline \multicolumn{3}{|l|}{ Inclusion criteria } \\
\hline \multicolumn{3}{|l|}{ Exclusion criteria } \\
\hline \multicolumn{3}{|l|}{$\begin{array}{l}\text { Method/s of recruitment of } \\
\text { participants }\end{array}$} \\
\hline Informed consent obtained & Yes/No/Unclear & \\
\hline Notes: & & \\
\hline
\end{tabular}

\section{Methods}

Descriptions as stated in report/paper Location in text

(pg \& fig/table)

\section{Aim/s of study}

Design (e.g. parallel, crossover)

Start date

End date

Copyright $\odot 2012$ The Cochrane Collaboration. Published by John Wiley \& Sons, Ltd. 
Total study duration

Ethical approval needed/ obtained for $\mathrm{Yes} / \mathrm{No} /$ Unclear study

Notes:

\section{Participants}

Provide overall data and, if available, comparative data for each intervention or comparison group.

Description as stated in report/paper
Location in text

(pg \& fig/table)

Total no. randomized

Baseline imbalances

Withdrawals and exclusions

(if not provided below in results section)

Age range

Sex

Severity of illness

(ARDS/ALI criteria, APACHE score, SOFA

score)

Co-morbidities

(if detailed)

Other treatment received (additional to study intervention)

Subgroups measured

Subgroups reported 
Notes:

5. Intervention groups

Copy and paste table for each intervention and comparison group

Intervention Group

Description as stated in report/paper

Location in text

(pg \& fig/table)

\section{Group name}

No. randomized to group

\section{Description of therapy}

- Type of delivery device [Vapotherm/Opti-

flow/other]

- Size of nasal cannula

- Litres/ minute delivered

\section{Duration of treatment period}

\section{Co-interventions}

Notes:

\section{Comparison Group I}

\section{Group name}

No. randomized to group 


\section{Description of therapy}

- Type of delivery device [Vapotherm/Opti-

flow/other]

- Size of nasal cannula

- Litres/ minute delivered

\section{Duration of treatment period}

\section{Co-interventions}

Notes:

Add another table if more than one comparison group

\section{Outcomes}

\section{Outcomes measures reported in paper (circle)}

Failure of treatment as indicated by the need for NIPPV or invasive Yes / No ventilation (up to 28 days)

In hospital mortality (up to 90 days)

Yes / No

\begin{tabular}{ll}
\hline Adverse events & Yes / No
\end{tabular}

Duration in hours of any form of respiratory support

Yes / No

(mechanical ventilation, NIPPV, HFNC, standard oxygen)

\begin{tabular}{l|l}
\hline Length of stay (ICU and hospital) & Yes / No
\end{tabular}

Degree of atelectasis on radiological examination

Yes / No

Positive end expiratory pressure measured at the pharyngeal level Yes / No $\left(\mathrm{cmH}_{2} \mathrm{O}\right)$

Oxygenation $\left(\mathrm{PaO}_{2} / \mathrm{FiO}_{2}\right.$ ratio, $\mathrm{PaO}_{2}, \mathrm{SaO}_{2}$ and $\left.\mathrm{SpO}_{2}\right)$

Yes / No

Carbon dioxide clearance $\left(\mathrm{PaCO}_{2}\right.$ and $\left.\mathrm{pCO}_{2}\right)$

Yes / No

Respiratory rate

Yes / No

Copyright $\odot 2012$ The Cochrane Collaboration. Published by John Wiley \& Sons, Ltd. 


\begin{tabular}{l|l} 
Work of breathing (joules per litre) & Yes / No \\
\hline Patient reported dyspnoea & Yes / No \\
\hline Patient reported comfort & Yes / No \\
\hline Patient reported mouth dryness & Yes / No \\
\hline Patient refusal to continue with treatment & Yes / No \\
\hline Cost comparison of treatment (in Australian dollars) & Yes / No \\
\hline
\end{tabular}

DETAILS OF OUTCOMES INCLUDED IN PAPER

Cut and paste for each included outcome

(Insert outcome name here)

\section{Description as stated in report/paper Location in text}

Outcome name as stated in paper

Unit of measurement

Time points measured

Time points reported

Person measuring/reporting

Is outcome/tool validated? $\quad$ Yes/No/Unclear

Missing data

Power

Notes:

\section{Results}

Copy and paste the appropriate table for each outcome, including additional tables for each time point and subgroup as required. Dichotomous outcome 


\section{Comparison group/s}

\section{Outcome}

\section{Subgroup}

\section{Timepoint \\ measured}

\begin{tabular}{|c|c|c|c|c|}
\hline \multirow{2}{*}{$\begin{array}{l}\text { Results } \\
\text { (add more compar- } \\
\text { ison groups here if } \\
\text { necessary) }\end{array}$} & \multicolumn{2}{|c|}{ Intervention } & \multicolumn{2}{|l|}{ Comparison } \\
\hline & No. events & No. participants & No. events & No. participants \\
\hline
\end{tabular}

No. missing participants and reasons

No. participants moved from other group/s and reasons

Any other results reported

Statistical methods used and appropriateness of these methods (e.g. adjustment for correlation)

Reanalysis required? (specify) $\quad$ Yes/No/Unclear

\begin{tabular}{l|l}
$\begin{array}{l}\text { Reanalysis } \\
\text { possible? }\end{array}$ & Yes/No/Unclear
\end{tabular}

Reanalysed results

Notes: 


\section{Comparison group/s}

\section{Outcome}

\section{Subgroup}

Timepoint measured

Post-intervention or

change from baseline?

\begin{tabular}{l|l|l|l|l}
\hline Results & Intervention & \multicolumn{3}{c}{ Comparison } \\
\cline { 2 - 5 } Mean & $\begin{array}{l}\text { SD (or other } \\
\text { variance) }\end{array}$ & $\begin{array}{l}\text { No. partici- } \\
\text { pants }\end{array}$ & $\begin{array}{l}\text { Sean (or other } \\
\text { variance) }\end{array}$ & $\begin{array}{l}\text { No. partici- } \\
\text { pants }\end{array}$ \\
\hline & & & \\
\hline
\end{tabular}

No. missing participants and reasons

No.

ticipants moved from other group and rea-

sons

Any other results reported

Statistical methods used and appropriateness of these methods (e.g. adjustment for correlation)

Reanalysis required? (specify) Yes/No/Unclear 
Reanalysis possible?

Yes/No/Unclear

Reanalysed results

Notes:

\section{Other information}

Description as stated in report/paper
Location in text

( $p g$ \& fig/table)

Key conclusions of study authors

References to other relevant studies

Correspondence required for further study information (from whom, what and when)

Notes:

Appendix 4. Quality Assessment Form

\section{Allocation of Intervention}

Comments

Method used to generate group allocation

Quality of group allocation $\quad$ Yes/No/Unclear

\section{Allocation Concealment}

Copyright @ 2012 The Cochrane Collaboration. Published by John Wiley \& Sons, Ltd. 
(Continued)

Method used to conceal allocation

\begin{tabular}{l|l}
\hline Quality of allocation concealment & Yes/No/ Unclear \\
\hline Blinding & Yes/No/Unclear \\
\hline Participant & Yes / No/Unclear \\
\hline Outcome assessor & Yes/No/Unclear \\
\hline \begin{tabular}{l} 
Other - Specify: \\
\hline $\begin{array}{l}\text { Intention-to-treat analysis was applied to } \\
\text { all } \\
\text { participants entering study }\end{array}$
\end{tabular} \\
\hline
\end{tabular}

$15 \%$ or fewer excluded

Not analysed as intention to treat

Unclear

Incomplete outcome data

Comments

Was outcome data complete?

\begin{tabular}{l|l}
\hline Primary Outcome & Yes/No/Unclear \\
\hline \begin{tabular}{l} 
Secondary Outcome 1 \\
\hline $\begin{array}{l}\text { Secondary Outcome } 2 \\
\text { (add more rows if necessary) }\end{array}$
\end{tabular} & Yes/No/Unclear \\
\hline
\end{tabular}

\section{Reporting bias}


Have all stated outcomes been fully reported?

\begin{tabular}{|c|c|}
\hline Primary Outcome & Yes/No/Unclear \\
\hline Secondary Outcome 1 & Yes/No/Unclear \\
\hline $\begin{array}{l}\text { Secondary Outcome } 2 \\
\text { (add more rows if necessary) }\end{array}$ & Yes/No/Unclear \\
\hline Other potential sources of bias & \\
\hline $\begin{array}{l}\text { Are there any other potential threats to va- } \\
\text { lidity? }\end{array}$ & \\
\hline $\begin{array}{l}\text { Imbalances of participants characteristics at } \\
\text { baseline } \\
\text { Crossover studies (Refer 16.4.3 in } \\
\text { Cochrane Handbook (Higgins 2011)) }\end{array}$ & $\begin{array}{l}\text { Yes/No/Unclear } \\
\text { Yes/No/Unclear }\end{array}$ \\
\hline $\begin{array}{l}\text { Other (Refer 8.15.1.5 in Cochrane Hand- } \\
\text { book (Higgins 2011)) }\end{array}$ & Yes/No/Unclear \\
\hline
\end{tabular}

\section{H I S T O R Y}

Protocol first published: Issue 11, 2012

\section{CONTRIBUTIONS OFAUTHORS}

Conceiving the review: Amanda Corley (AC)

Co-ordinating the review: AC

Undertaking manual searches: AC

Screening search results: AC, Claire Rickard (CR), Leanne Aitken (LA), Amy Johnston (AJ)

Organizing retrieval of papers: AC

Screening retrieved papers against inclusion criteria: AC, CR, LA, AJ

Appraising quality of papers: AC, CR LA, AJ

High flow nasal cannulae for respiratory support in adult intensive care patients (Protocol)

Copyright @ 2012 The Cochrane Collaboration. Published by John Wiley \& Sons, Ltd. 
Abstracting data from papers: AC, CR, LA, AJ

Writing to authors of papers for additional information: AC

Providing additional data about papers: AC

Obtaining and screening data on unpublished studies: AC, CR, LA, AJ

Data management for the review: AC

Entering data into Review Manager (RevMan 5.1): AC

RevMan statistical data: Adrian Barnett, AC

Other statistical analysis not using RevMan: Adrian Barnett (AB), AC

Interpretation of data: AC, John Fraser (JF), CR, LA, AJ

Statistical inferences: $\mathrm{AC}, \mathrm{AB}, \mathrm{CR}$

Writing the review: AC, CR, LA, AJ, AB, JF

Securing funding for the review: AC

Performing previous work that was the foundation of the present study: AC, JF, AB

Guarantor for the review (one author): AC

Person responsible for reading and checking review before submission: AC

\section{DECLARATIONSOF INTEREST}

JF has received an unrestricted grant from Fisher and Paykel Healthcare Ltd, the makers of Optiflow which is a high flow nasal cannulae delivery system. This grant assisted in partially funding the employment of a research nurse to complete the study 'Oxygen delivery through high-flow nasal cannulae increases end expiratory lung volume and reduces respiratory rate in post-cardiac surgical patients when compared to standard low flow oxygen' (Corley 2011). Fisher and Paykel Healthcare Ltd had no part in the study design, data collection, data analysis or manuscript preparation.

Fisher and Paykel Healthcare Ltd have paid for AC to attend the American Thoracic Society meeting in New Orleans, May 2010 (airfare only) and a Fisher and Paykel research meeting in Auckland, New Zealand, August 2009 (airfare and accommodation). JF has received travel funding from Fisher and Paykel to speak at research meetings in Paris (2009) and London (2010) on the Critical Care Research Group's respiratory research program, which included the study mentioned above.

$\mathrm{AC}, \mathrm{AB}$ and JF are investigators for a study which is currently underway (Corley, in progress) and which may be eligible for inclusion in the review. This study is supported by a grant from The Prince Charles Hospital Foundation.

Authors CR, L A and AJ have no conflicts of interest to declare.

\section{SOURCES OF SUPPORT}




\section{Internal sources}

- No sources of support supplied

\section{External sources}

- Australian College of Critical Care Nurses Systematic Review Grant 2011, Australia. 